\title{
Determination of Total IgE by ELISA in Tubes and Plates Compared with PRIST
}

\author{
NANCY K. BAYNE and KENNETH P. MATHEWS \\ Division of Allergy, Department of Internal Medicine \\ University of Michigan Medical School, Ann Arbor, Michigan, USA, 48109
}

(Accepted April 20, 1982)

\begin{abstract}
$\operatorname{IgE}$ was measured by ELISA in tubes and in microtiter plates. the results being compared with PRIST data. The recommended readings of the tube contents in a spectrophotometer (SPM) were compared with results using a multi-channel photometer (MCP).

Geometric mean values (International Units) and standard deviations of $\mathbf{4 4}$ normal sera examined by the 3 different methods were: PRIST 16.2 \pm 4.0 ; SPM 15.6 \pm 4.9 and MCP 18.4 \pm 4.4 . Correlation coefficients were: $P R I S T$-SPM $r=0.98 ;$ PRIST-MCP $r=0.98$; and SPM-MCP $r=0.97$. Intra- and inter-assay coefficients of variation were smaller for MCP than for SPM. In addition, reading in microtiter plates was much faster, while having little effect on sensitivity.
\end{abstract}

IN RECENT YEARS IGE HAS BEEN MEASURED in most laboratories (including our own) by the paper radioimmunosorbent test (PRIST) (1). Due to recent advances in methodology of the enzyme-linked immunosorbent assay (ELISA) technique (2) as well as a desire to reduce radioactive waste and exposure, a study was undertaken to evaluate a commercially available ELISA test kit. This study sought to compare results of ELISA measurements of total $\mathrm{IgE}$ in tubes versus microtiter plates as well as to provide further data comparing results with PRIST measurements.

\section{Materials and Methods}

\section{PRIST assays:}

Phadebas IgE PRIST kits were purchased from Pharmacia Diagnostics, Piscataway, NJ 02052, USA (product number 611961 . The procedure is detailed in the product information. Anti-IgE covalently-coupled paper dises were allowed to incubate with the test sera or standards. After washing, the discs were incubated overnight with $100 \mu$ of ${ }^{125} \mathrm{I}$-anti IgE. Unbound radioactivity was removed by washing, and radioactivity of the ${ }^{125} \mathrm{I}$-anti-IgE bound to the test serum IgE was then measured in a gamma counter.

\section{ELISA measurements:}

The "Enzygnost IgE" kit is product number 967361 of the Calbiochem-Behring Corporation, La Jolla, CA 92037, USA. Following product instructions, test specimens were incubated in tubes coated with goat anti-human IgE following which peroxidase-labelled rabbit anti-human IgE, enzyme substrate and sulfuric acid were successively added. The tubes were read in a spectrophotometer (SPM) at a wavelength of $492 \mathrm{~nm}$, as recommended. In addition, samples were read on a multi-channel

Correspondence: Kenneth P. Mathews R6621 Kresge Medical Research Bldg. I University of Michigan Medical Center Ann Arbor, Michigan 48109 photometer (MCP). In order to read samples in this way, 280 $\mu$ l of each final reaction mixture was transferred to the wells of microtiter plates. The plate was read approximately $25 \mathrm{~min}$ after stopping the reaction.

Test sera:

Forty four normal adult sera were evaluated by the three different methods. Individuals were asked to complete brief questionnaires relating to history of allergies. Only sera from those with all negative replies to a history of asthma, hayfever. eczema, atopic dermatitis and other allergies were included in the study.

\section{EXPERIMENTS AND RESULTS:}

The geometric mean results (International units) and standard deviations of the forty-four sera examined by the three different methods were:

Method

Geometric Mean

Standard

Deviation*

$\begin{array}{lll}\text { PRIST } & 16.2 \mathrm{IU} / \mathrm{ml} & 4.0 \\ \text { ELISA - SPM } & 15.6 \mathrm{IU} / \mathrm{ml} & 4.9 \\ \text { ELISA - MCP } & 18.4 \mathrm{IU} / \mathrm{ml} & 4.4\end{array}$

Correlation coefficients were: PRIST-SPM $r=0.98$; PRIST-MCP $r=0.98$; and SPM-MCP $r=0.97$, as seen in Table 1. Comparison of individual values obtained by the two methods of reading the ELISA assay is illustrated in Fig. 1.

In addition to the analysis with normal serum, six different patient serum samples were assayed by each of the three methods. These samples ranged from about 3 to $3000 \mathrm{IU} \mathrm{IgE/ml.} \mathrm{Six} \mathrm{analyses} \mathrm{of} \mathrm{each} \mathrm{of} \mathrm{the} \mathrm{six} \mathrm{patients'}$ sera were performed within a single assay run. The mean intra-assay coefficient of variation (CV) of these six different serum samples was $4.8 \%$ when transferred to microtiter plates and read on the multi-channel photometer and $7.5 \%$ when read by the recommended procedure on the spectrophotometer.

The CV between these samples on three different runs was $9.6 \%$ with MCP and $12.1 \%$ with SPM. The CV was not systematically affected by the level of IgE (see Table 2).

In addition to the analysis with six different patient sera, a single kit-supplied control was run in 10 different

*These S.D., based on the anti-logs of the transformed values, are shown for comparative purposes but are not intended to reflect the much larger confidence intervals used clinically. 


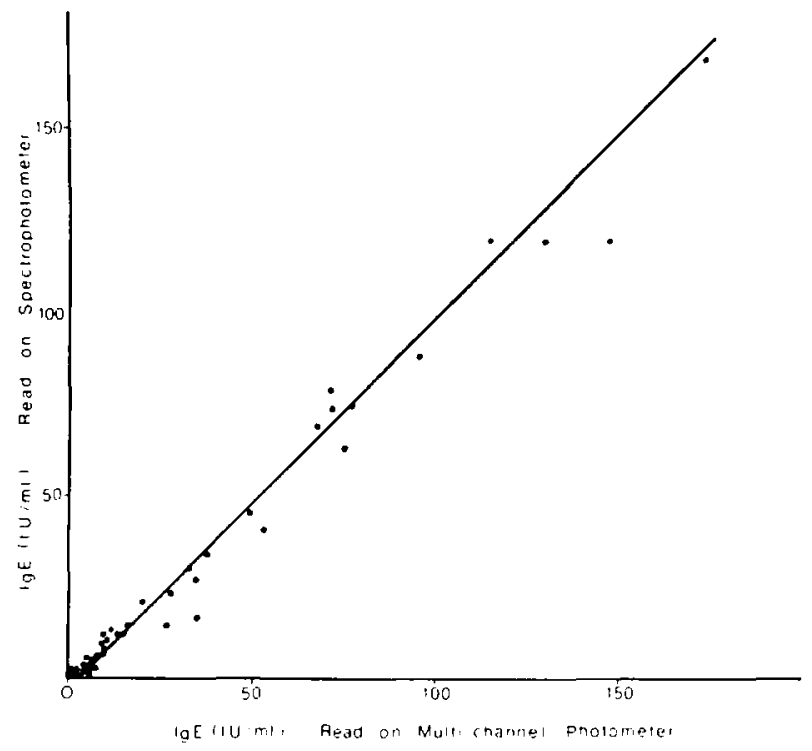

Fig. 1 - Normal IgE values. Correlation of ELISA-MCP and ELISA-SPM.

assays using the spectrophotometer and in 10 different assays during the multi-channel photometer. The mean value of this control serum was 87.80 IU with a standard deviation of 7.38 when read on the MCP, yielding an interassay CV of $8.4 \%$; the mean value of the same sample using the SPM was 88.60 with a standard deviation of 10.35 and an inter-assay $\mathrm{CV}$ of $11.7 \%$.

The detection limit of the ELISA assay using MCP and SPM was calculated as the smallest single result which, with a stated probability, could be distinguished from a suitable blank. Using the mean blank result \pm 2.5 standard deviations, the detection limit was calculated to be $0.48 \mathrm{IU} / \mathrm{ml}$ using the MCP and $0.22 \mathrm{IU} / \mathrm{ml}$ using SPM. The sensitivity limit also was determined experimentally by preparing serial two-fold dilutions of individual subjects' sera down to the point where they no longer gave readings greater than the blank +2.5 standard deviations. Then the sensitivity limit is the concentration in the most dilute tube of the series which can be read above this detection limit. The mean values obtained for two different individuals were $0.50 \mathrm{IU} / \mathrm{ml}$ by $\mathrm{MCP}$ and 0.34 IU/ml by SPM.

\section{Discussion}

Due to the normally very low levels (nanograms/ml) of total $\mathrm{IgE}$ in human serum, the technique used to measure IgE must be very sensitive. The initial solid phase inhibition radioimmunoassay for IgE (RIST) (3) gave falsely high values at low IgE levels, but results by PRIST are highly satisfactory (4). However, recently the ELISA technique has been found to be applicable in many areas (2). It has as its major advantage the fact that no radioactive materials are involved. Consequently, the problems of decay of assay materials, exposure to radiation and disposal of radioactive wastes are eliminated. It does not require expensive equipment and is relatively simple to carry out. At the same time the enzyme method offers degrees of sensitivity and specificity comparable to the radioimmunoassay. Application of the ELISA principle to the measurement of serum $\operatorname{IgE}$ actually was proposed a decade ago by Hoffman (5) and
TABLE 1

Correlation Coefficients Comparing The Different Methods

\begin{tabular}{lr}
\hline \hline Methods Compared & r Value \\
PRIST.ELISA (SPM) & 0.98 \\
PRIST.ELISA (MCP) & 0.98 \\
ELISA (SPM) - ELISA (MCP) & 0.97
\end{tabular}

TABLE 2

COEFficients of VARIATION COMPaRING ELISA READ ON A SPECTROPHOTOMETER (SPM) AND ELISA READ ON A MULTI ChanNel Photometer (MCP)

\begin{tabular}{lcc}
\hline & CoEfFicient of VARIATIO \\
Method & Intra-assay & Inter-assay \\
ELISA - MCP & & \\
ELISA - SPM & 4.8 & 9.6 \\
\end{tabular}

subsequently confirmed by others $(6)$, but the recent introduction of a commercially available kit for this purpose has stimulated increased interest in this procedure.

In this study we have compared results of ELISA measurements of $\mathrm{IgE}$ in tubes versus microtiter plates as well as comparing results with PRIST data. From the data presented, it appears that the ELISA method yields total IgE values very similar to those obtained from the well-accepted PRIST radioimmunoassay. Our adaptation of the ELISA to reading in a multi-channel photometer by transfer of final reaction mixture to microtiter plates increases the ease and efficiency of obtaining results. Geometric mean values obtained using this adaptation correlate well with those obtained following recommended procedures, and the standard deviations obtained by evaluating forty-four normal sera were comparable when either the ELISA-SPM, ELISA-MCP, or PRIST methods were used. Likewise the ELISA-MCP results correlated very closely with the ELISA-SPM and PRIST results.

In addition, ELISA-MCP showed lower CV values in both inter-assay and intra-assay comparisons. It is to be expected that intra-assay variations would be less when microtiter plates are used, as all samples are read within a time period of approximately $2 \mathrm{~min}$. When reading 50 to 100 samples on the conventional spectrophotometer, it may take up to 50-100 min, which allows time for some minimal color change between the first and last sample read. We found lower $C V$ values with use of the multichannel photometer both with sera having a wide range of $\operatorname{IgE}$ levels and with the control supplied in the kit when using the Enzygnost ELISA method.

As more physicians become familiar with the use (and limitations) of serum IgE measurements, requests for these determinations are increasing. Elevated values are commonly found in parasitic diseases, atopic diseases (allergic asthma, rhinitis, eczema and urticaria), bullous pemphigoid, some cases of bronchiolitis, bron. chopulmonary aspergillosis, rare cases of myeloma, liver disease, and some types of immune deficiency disease (Wiskott-Aldrich syndrome, hyper-IgE syndrome, DiGeorge syndrome, Nezelof syndrome and others) $(7,8)$. The latter instances are thought to arise from a deficien- 
cy of suppressor $\mathrm{T}$ cells regulating $\mathrm{Ig} \mathrm{E}$ formation, but IgE may be essentially absent in other types of immunodeficiency (eg. ataxia telangiectasia, X-linked agammaglobulinemia). Measurements of serum IgE may help to differentiate the above diagnoses from similar conditions, but the results have to be interpreted in the light of other clinical and laboratory data. There also is evidence that elevated serum IgE levels in young children are predictive of the subsequent development of allergic disease (9). It also is important to compare results in children with normals for the same age, since $\mathrm{IgE}$ levels gradually rise from an almost undetectable level at birth to maximal levels by age 10 years (10).

From the various analyses performed, we conclude that the IgE measurements by ELISA correlate very well with the PRIST assays. ELISA is sensitive and reproducible, and when read in microtiter plates, it is much faster and improves replicability while having little effect on sensitivity. In addition to the above advantages, this procedure eliminates decay of assay materials, radiation exposure and radioactive waste disposal.

\section{REFERENCES}

1. Ceska, M. and Lundkrist. U. A new and simple radioim munoassay method for the determination of $\mathrm{IgE}$. Immunochemistry 9, 1021-1030 (1972).

2. O'Sullivan, M.J., Bridges, J.W., and Marks, V. Enzyme immunoassay: a review. Ann. Clin. Biochem. 16, 221-240 (1979).

3. Johansson, S.G.O., Bennich, H.H., and Wide, L. A new class of immunoglobulin in human serum. Immunology 14 , 265-272 (1968).

4. Johansson, S.G.O. Berglund, A. and Kjellman, N.I.M. Comparison of $\operatorname{IgE}$ values as determined by different solid phase radioimmunoassay methods. Clin. Allergy 6, 91-98 (1976).

5. Hoffman, D.R. Estimation of serum IgE by an enzyme linked immunosorbent assay (ELISA). J. Allergy Clin. Immunol 51, 303-307 (1973).

6. Weltman. J.K., Fracketon, A.R., Szaro, R.P. and Rotman, B. A galactosidase immunosorbent test for human immunoglobulin E. J. Allergy Clin. Immunol. 58, 426-431 (1976).

7. Heiner, D.C. and Rose, B. Elevated levels of $\partial \mathrm{E}(\mathrm{IgE})$ in conditions other than classical allergy. J. Allergy $4 \mathbf{5}, 30-42$ (1970).

8. Parker, C.W. Clinical Immunology. Philadelphia, WB Saunders, (1980). Pp. 1387-1390.

9. Kjellman, N.I.M. Predictive value of high $\operatorname{IgE}$ levels in children. Acta Paediatr. Scand. 65, 465-471 (1976).

10. Kjellman, N.I.M., Johansson, S.G.O. and Roth, A. Serum IgE levels in healthy children quantified by a sandwich technique (PRIST). Clin. Allergy 6. 51-59 (1976). 